\title{
Advances in Isotope Ratio Mass Spectrometry and Required Isotope Reference Materials
}

\author{
Jochen Vogl \\ BAM Federal Institute for Materials Research and Testing, Unter den Eichen 87, 12205 Berlin, Germany
}

\begin{abstract}
The article gives a condensed version of the keynote lecture held at the International Mass Spectrometry Conference 2012 in Kyoto. Starting with some examples for isotope research the key requirements for metrologically valid procedures enabling traceable and comparable isotope data are discussed. Of course multi-collector mass spectrometers are required which offer sufficiently high isotope ratio precision for the intended research work. Following this, corrections for mass fractionation/ discrimination, validation of the analytical procedure including chemical sample preparation and complete uncertainty budgets are the most important issues for obtaining a metrologically valid procedure for isotope ratio determination. Only the application of such metrologically valid procedures enables the generation of traceable and comparable isotope data. To realize this suitable isotope and/or $\delta$-reference materials are required, which currently are not sufficiently available for most isotope systems. Boron is given as an example, for which the situation regarding isotope and $\delta$-reference materials is excellent. Boron may therefore serve as prototype for other isotope systems.
\end{abstract}

Keywords: isotope reference materials, delta reference materials, traceability, comparability, measurement uncertainty, synthetic isotope mixtures, mass fractionation, mass discrimination

(Received September 6, 2012; Accepted December 17, 2012)

\section{INTRODUCTION}

The isotopic compositions of specific elements vary in nature mainly because of radioactive decay, mass dependent fractionation and isotope effects in chemical reactions. ${ }^{1)}$ These effects are known for decades and a broad field of applications has been established ranging from geochemistry to nuclear forensics and archaeometry. ${ }^{2-4)}$ Improvement of the analytical procedures and of the mass spectrometric instrumentation led to an extension of the application field to the biosphere, for example plant metabolism and food authenticity. ${ }^{5,6)} \mathrm{A}$ good example for the latter is the use of the ${ }^{87} \mathrm{Sr} /{ }^{86} \mathrm{Sr}$ isotope ratio for determining the geographic origin of Emmental cheese by Fortunato et al. ${ }^{7)} \mathrm{The}^{87} \mathrm{Sr} /{ }^{86} \mathrm{Sr}$ isotope ratio varies in minerals and rocks due to the initial $\mathrm{Rb}$ concentration and the time passed by since formation of the mineral/rock. This variation is reflected in the soil derived from the minerals and bedrock and may be influenced by water and atmospheric input. Grass which is grown on this soil also reflects this ${ }^{87} \mathrm{Sr} /{ }^{86} \mathrm{Sr}$ isotope ratio and also the milk of pastured cows, if no $\mathrm{Sr}$ containing supplement is being fed. Therefore, cheese made from the milk of pastured cows should also reflect the ${ }^{87} \mathrm{Sr} /{ }^{86} \mathrm{Sr}$ isotope ratio of the soil-rocksystem. As a result Fortunato et al. could show that Emmental cheese from regions showing different geochemical background such as Finland and Australia are distinguishable from Swiss Emmental cheese, whereas samples from Allgäu, an alpine part of southern Germany, are indistinguishable from Swiss Emmental cheese, because of similar geochemical background. ${ }^{7}$

Beside these natural isotope variations, man-made isotope shifts are of interest to an even wider range of

Correspondence to: Jochen Vogl, BAM Federal Institute for Materials Research and Testing, Unter den Eichen 87, 12205 Berlin, Germany, e-mail: jochen.vogl@bam.de applications. Here artificially enriched isotopes are used to create such effects. In technique for example ${ }^{10} \mathrm{~B}$ is used for neutron shielding in nuclear applications, due to its extremely high cross section for neutron absorption. ${ }^{8)}$ Apart from this, boron isotope compositions have been used for classical geo- and cosmochemical research (e.g. paleo $\mathrm{pH}$ modelling of the oceans via marine carbonates ${ }^{9)}$ or contamination studies in the hydrosphere ${ }^{10)}$ ) and to determine the origin of food products. ${ }^{11)}$

Enriched isotopes of dietary minerals, e.g. $\mathrm{Mg}$, Ca or $\mathrm{Fe}$, are used as so-called tracer in nutrition physiology to unravel metabolism and pathways of these particular elements. ${ }^{12-14)}$ In isotope dilution mass spectrometry the enriched isotope is used as so-called spike for the quantification of element or species concentrations. ${ }^{15,16)}$

All isotope research, whether investigation of natural isotopic variations or investigation of man-made isotope shifts, requires the determination of one or more isotope ratio typically carried out by mass spectrometric techniques. Currently, for elements other than noble gases and classical stable isotopes $(\mathrm{H}, \mathrm{C}, \mathrm{N}, \mathrm{O}, \mathrm{S})$ the most important mass spectrometric techniques for isotope ratio determinations are thermal ionization mass spectrometry (TIMS), inductively coupled plasma mass spectrometry (ICPMS) and secondary ionization mass spectrometry (SIMS). All these mass spectrometric techniques are affected by mass fractionation/ discrimination, which results in a bias of the observed isotope ratio. ${ }^{17,18)}$ This bias, however, depends on the type of instrument and the adjustable instrument parameters. In order to obtain comparable isotope data between different instrumental techniques and laboratories it is necessary to correct for these effects. The most important strategies are listed in the following:

Correction factors, also called K-factors, are obtained by dividing the certified isotope amount ratio by the observed isotope ratio of an isotope reference material and applying this K-factor to the subsequent sample determination under 


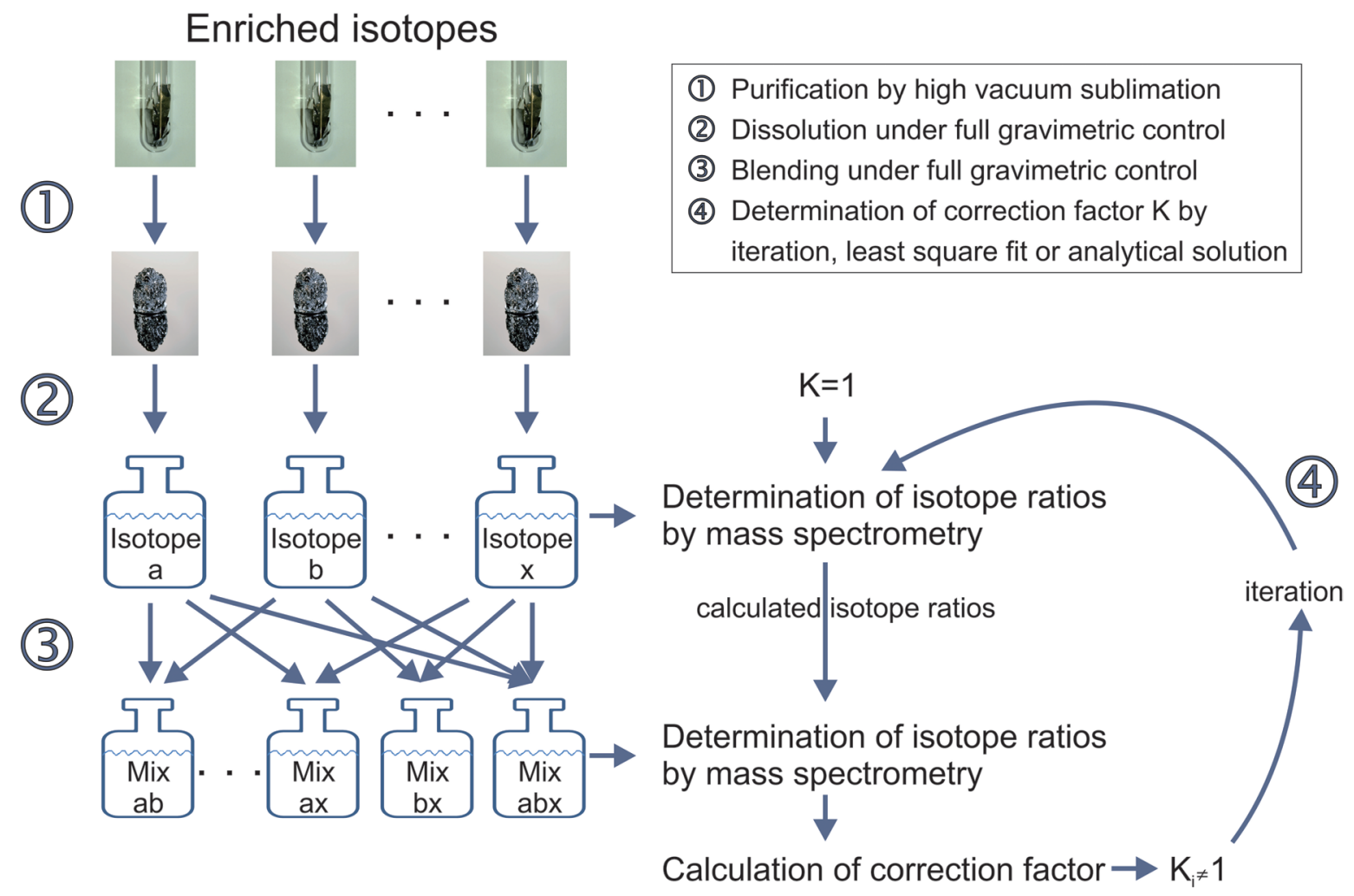

Fig. 1. Schematic principle of the synthetic isotope mixture approach for correcting instrumental mass fractionation/discrimination by full calibration of the mass spectrometer. Copied with permission. Copyright BAM 2012.

the same analytical conditions. ${ }^{19)}$

Mass discrimination coefficients are obtained for a specific isotope ratio of an element, which is considered as being constant, and are applied to the correction of another isotope ratio of this element. This correction often is referred to as internal correction and is applied for example to ${ }^{87} \mathrm{Sr} /{ }^{86} \mathrm{Sr}$ determinations. ${ }^{7}$

The double spike technique is a specific case of an internal correction, where 2 of at least 4 isotopes of an element are enhanced by adding a so-called double-spike consisting of 2 enriched isotopes. With knowledge of the double-spike composition it is possible to obtain the corrected isotope ratios by an iterative calculation approach. ${ }^{20)}$

Synthetic isotope mixtures are prepared by highly enriched and highly purified isotopes and are used to obtain the correction factors for each isotope ratio by an iterative calculation. $^{21,22)}$ Although being expensive in labour and money, this approach is still the best route to achieve "true" or absolute isotope amount ratios with smallest uncertainties for atomic weight determination and certification of isotope reference materials. The schematic principle of the synthetic isotope mixture approach is given in Fig. 1.

In relative measurements the observed isotope ratio of the sample is related to the observed isotope ratio of a commonly accepted standard and the difference, the so-called $\delta$-value, is expressed in per mill. ${ }^{23,24)} \mathrm{By}$ this calculation the correction for mass fractionation/discrimination cancels down and does not have to be determined. Usually, $\delta$-value can be determined more precise than "true" or absolute isotope amount ratios. ${ }^{25)}$

The last approach for dealing with mass fractionation effects is total evaporation, which is restricted to TIMS. ${ }^{17)}$
Here the entire sample is evaporated and the ion intensities are integrated over the whole time. By doing this mass fractionation effects are minimized and only a small remaining part has to be corrected for, which leads to an improved repeatability. ${ }^{26)}$

Beside the correction of instrumental mass fractionation the chemical sample preparation is equally important. Especially, inappropriate contamination, mass fractionation effects and incomplete matrix separation during sample preparation are difficult to correct for. These sources of bias or even error are specific for each analyte-matrix combination and therefore the validation of the analytical procedure should pay particular attention to this for every analytematrix combination. ${ }^{11)}$

To obtain state-of-the-art isotope data multi-collector instruments are needed, which offer sufficient precision, metrologically valid procedures enabling the estimation of the measurement uncertainty and isotope reference materials for calibration and validation.

\section{MC-ICPMS AND MC-TIMS}

The current generation of MC-ICPMS and MC-TIMS has reached a very high level in the isotope ratio performance. The MC-ICPMS instruments $\mathrm{Nu}$ Plasma II ( $\mathrm{Nu}$ Instruments, Wrexham, UK) and Neptune Plus (Thermo Fisher Scientific, Bremen, DE) offer specified parameters such as high resolution capabilities (mass resolving power $>7000$ ) which enable the separation of plasma based interferences, external reproducibilities expressed as relative standard deviations (RSD) for ${ }^{87} \mathrm{Sr} /{ }^{86} \mathrm{Sr}$ determinations of $<0.002 \%$ and sensitivities that allow precise isotope ratios in the low $\mathrm{ng} / \mathrm{g}$ 
concentration range. ${ }^{27,28)}$ The MC-TIMS instruments Phoenix (Isotopx, Middlewich, UK) and Triton (Thermo Fisher Scientific, Bremen, DE) specify external reproducibilities for ${ }^{87} \mathrm{Sr} /{ }^{86} \mathrm{Sr}$ determinations even at $0.0005 \%$ (RSD). ${ }^{29,30)}$ Instruments from the preceding MC-ICPMS or MC-TIMS generation such as the IsoProbe (formerly GV Instruments) enable similar external reproducibilities down to $0.003 \%$ (relative standard deviation of the sampling distribution, RSE) as demonstrated for ${ }^{112} \mathrm{Cd} /{ }^{111} \mathrm{Cd}$ by Pritzkow et al. ${ }^{31)}$ An overview of currently achievable precision in isotope ratio determination for selected elements, so-called nontraditional stable isotopes, is given in Table 1. As expected lighter elements such as magnesium, copper and zinc show slightly lower precision than heavier elements such as cadmium. Surprisingly the instrument type and the calibration type play no significant role for the achieved precision in MC-ICPMS measurements. In the case of Cd "Double spike with Nu Plasma," "Ag doping with Axiom" and "observed data with IsoProbe" show similar precision. But the "TIMS double spike technique" yields a precision which is higher by a factor of 4 , which is also supported by the higher precision of TIMS specifications for ${ }^{87} \mathrm{Sr} /{ }^{86} \mathrm{Sr}$ determinations (see above). One reason for this systematical difference between TIMS and MC-ICPMS could be the slow response of the Faraday amplifier in combination with ion source characteristics. In ICPMS the signal intensity can vary or oscillate due to the enclosure of air in ICP, pressure oscillation of the expansion pressure as well as introduction of large-sized solution mist into the ICP which may cause signal spikes. In TIMS, however, changes in the signal intensity occur on much longer scale than those in ICPMS. Therefore, the slow response does not affect the precision of TIMS, but those for ICPMS. More details on this topic can be obtained from ref. 32.

When discussing the precision values in Table 1 it has to be noted that these data have been obtained on Cd reference materials or standard solutions. Isotope ratio determinations in real samples usually result in higher precision values due to analyte and matrix inhomogeneities in the sample and artefacts caused by sample preparation and analyte separation. Therefore, careful investigation of all analytical steps and validation of the whole procedure by using isotope reference materials is indispensible.

\section{METROLOGICAL BACKGROUND}

When isotope data of different sources, e.g. from different publications or different laboratories, are conjointly used for calculations, modelling or any comparison purpose, all data have to be metrologically comparable, which in turn requires for all data to be metrologically traceable to the same reference. ${ }^{38)}$ Metrological traceability is "the property of a measurement result, whereby the result can be related to a reference through a documented unbroken chain of calibrations, each contributing to the measurement uncertainty." ${ }^{38)}$ Such reference can be an international standard or unit (e.g., international system of units-SI) or an isotope reference material, as it is the case for many $\delta$-values (e.g., NIST SRM 951 for $\left.\delta^{11} \mathrm{~B}\right)$. Additionally an uncertainty contribution for each calibration, which can be a correction factor or any other quantity contributing to the measurement result, has to be considered in the combined uncertainty of the measurement result. The measurement uncertainty itself is defined as a "non-negative parameter characterizing the dispersion of the quantity values being attributed to a measurand, based on the information used." ${ }^{38)}$ Thus the measurement uncertainty defines the interval, within which the measurement result is regarded as accurate or in other words as precise and true. The reproducibility of a measurement result, however, only provides information on the precision, but not on the trueness of the result and therefore pure reproducibility gives no information on the accuracy of a result (for more details see ref. 39).

In isotope analysis measurement uncertainty and traceability are no common knowledge and only a few scientists implement them in their daily work. Admittedly, uncertainty is mentioned in many publications, but in most cases the reproducibility is simply renamed and often it is not clearly stated, whether (sample) standard deviation (abbr. $s$ ) or standard deviation of the sampling distribution (abbr. $S E$ ), also named standard error (of the mean: $S E=s / \sqrt{n}$ ), is used. To illustrate the calculation of an uncertainty budget an example for boron will be given in the following. $\delta^{11} \mathrm{~B}$ values usually are obtained by measuring the sample isotope ratio ${ }^{11} \mathrm{~B} /{ }^{10} \mathrm{~B}$ versus those of NIST SRM 951 and calculating the $\delta^{11} \mathrm{~B}$ according Eq. (1). ${ }^{40)}$ For the uncertainty budget, however, we need to consider all potential influences on the measured quantities as shown by Rosner et al. for $\delta^{11} \mathrm{~B}$ determinations. ${ }^{11)}$ According to this work influences from

Table 1. Isotope ratio determinations and achievable precision obtained by different mass spectrometric techniques; precision is obtained on reference materials or standard solutions.

\begin{tabular}{|c|c|c|c|c|c|c|}
\hline \multirow{2}{*}{ Isotope ratio } & \multicolumn{2}{|c|}{ Precision * } & \multirow{2}{*}{ Calibration } & \multicolumn{2}{|c|}{ Instrument } & \multirow{2}{*}{ Ref. } \\
\hline & Rel. value & Type** & & Category & Type & \\
\hline${ }^{25} \mathrm{Mg} /{ }^{24} \mathrm{Mg}$ & $0.009 \%$ & $2 \sigma$ & Bracketing & MC-ICPMS & Nu Plasma & 33 \\
\hline${ }^{26} \mathrm{Mg} /{ }^{24} \mathrm{Mg}$ & $0.014 \%$ & $2 \sigma$ & Bracketing & MC-ICPMS & Nu Plasma & 33 \\
\hline${ }^{65} \mathrm{Cu} /{ }^{63} \mathrm{Cu}$ & $0.005 \%$ & $2 s$ & Ni doping, bracketing & MC-ICPMS & Neptune & 34 \\
\hline${ }^{66} \mathrm{Zn} /{ }^{64} \mathrm{Zn}$ & $0.005 \%$ & $2 s$ & ${ }^{64} \mathrm{Zn}-{ }^{67} \mathrm{Zn}$ double spike & MC-ICPMS & Neptune & 34 \\
\hline${ }^{110} \mathrm{Cd} /{ }^{112} \mathrm{Cd}$ & $0.0014 \%$ & $2 S D$ & ${ }^{106} \mathrm{Cd}-{ }^{108} \mathrm{Cd}$ double spike & MC-TIMS & Triton & 35 \\
\hline${ }^{114} \mathrm{Cd} /{ }^{110} \mathrm{Cd}$ & $<0.007 \%$ & $2 S D$ & ${ }^{111} \mathrm{Cd}-{ }^{113} \mathrm{Cd}$ double spike & MC-ICPMS & Nu Plasma & 36 \\
\hline${ }^{114} \mathrm{Cd} /{ }^{110} \mathrm{Cd}$ & $0.005 \%$ & $2 s$ & Ag doping, bracketing & MC-ICPMS & Axiom & 37 \\
\hline${ }^{112} \mathrm{Cd} /{ }^{111} \mathrm{Cd}$ & $<0.007 \%$ & $2 s_{\text {mean }}$ & None, observed data & MC-ICPMS & IsoProbe & 31 \\
\hline
\end{tabular}

* Precision on repeated measurements of the same sample/standard solution.

** as noted in the corresponding publication, in case the one fold value is given in the publication it has been multiplied by 2 to achieve the same basis; $\sigma, s$ and SD are assumed to be relative standard deviations and $s_{\text {mean }}$ is the relative standard deviation of the mean or the relative standard error. 
sample decomposition, boron-matrix separation, procedure blank, potential inhomogeneity of the $\delta=0$ reference material and the reproducibility of the isotope ratio determined in the reference material have to be considered beside the reproducibility of the sample isotope ratio determination. The isotope ratios of sample and reference material are already included in Eq. (1). To include the other influence quantities Eq. (1) has to be expanded to Eq. (2). ${ }^{11)}$

$$
\begin{gathered}
\delta^{11} \mathrm{~B}=\left(\frac{R_{\text {Sample }}}{R_{\text {NIST951 }}}-1\right) \times 1000 \\
\delta^{11} \mathrm{~B}=\left(\left(\frac{\left(R_{\text {Sample }} \cdot \kappa_{1} \cdot \kappa_{2} \cdot \kappa_{3}\right)}{\left(R_{\text {NIST } 951} \cdot \kappa_{4}\right)}\right)-1\right) \times 1000
\end{gathered}
$$

Reprinted with permission from ref. 11. Copyright 2011 American Chemical Society.

Here $\kappa_{1}, \kappa_{2}, \kappa_{3}$, and $\kappa_{4}$ are added as factor to the quantity they influence. The value of $\kappa_{1}, \kappa_{2}, \kappa_{3}$, and $\kappa_{4}$ is 1 in each case, because an influence could not be determined with the applied techniques. The attributed uncertainties for $\kappa_{1}, \kappa_{2}$, $\kappa_{3}$, and $\kappa_{4}$ derive from estimations or worst case scenarios. With these data the uncertainty budget for $\delta^{11} \mathrm{~B}$ determination can be calculated (Table 2), either by self-made spreadsheets or by specific software such as GUM-Workbench (Metrodata $\mathrm{GmbH}$, Weil am Rhein, Germany) both following ISO/BIPM ${ }^{41)}$ and EURACHEM/CITAC ${ }^{42)}$ guidelines. Quantities deriving from a measurement distribution are type A and the standard uncertainty can be taken as standard error of the mean. All other quantities are type B and the standard uncertainty derives from previous uncertainty calculations such as certificates, combined uncertainties or expert judgment. The combined standard uncertainty, $u_{c}$ $(k=1)$, for the $\delta^{11} \mathrm{~B}$-value is significantly larger than the reproducibility, either expressed as single standard deviation or standard error, because additional sources of uncertainty have been considered. Commonly the measurement result is expressed with the expanded uncertainty, $U=k \cdot u_{\mathrm{c}}$ with $k=2$, which gives in this case a $\delta^{11} \mathrm{~B}$-value of $(-23.8 \pm 1.2) \%$ for the "White Cabbage" reference material BCR-679.

Measurement uncertainties are necessary to decide whether results from the same or from different data sets are compatible or not. Two results are metrologically compatible, if and when the difference is smaller than the expanded uncertainty of the difference. ${ }^{38)}$ When only precision is provided this assessment is not possible, especially for data from different datasets, because the results are not obtained under repeatability conditions and may contain different sources of uncertainty.

In addition a complete uncertainty budget is useful for validating an analytical procedure, which is prescribed by ISO 17025 for all laboratory-developed or adapted procedures. ${ }^{43)}$ Validation confirms by experimental proof that the analytical procedure is suitable for the analytical task; in particular performance parameters such as measurement uncertainty, detection limit, selectivity, precision and robustness shall demonstrate the suitability for the intended use. This confirmation can be achieved by one of, or a combination of the following techniques:

1) calibration using reference materials;

2) comparison of results achieved with independent methods;

3) interlaboratory comparisons;

4) systematic assessment of all influencing quantities;

5) assessment of the measurement uncertainty, which is based on scientific understanding and practical experience.

Reference materials in isotope analysis are available for approximately $50 \%$ of the elements with two or more isotopes, ${ }^{25)}$ which in turn limits technique 1 to $50 \%$ of the stable isotope systems. Technique 2 and 3 are difficult to realize, because in isotope analysis (excepting $\mathrm{U}$ and $\mathrm{Pu}$ ) really independent procedures do hardly exist and interlaboratory comparisons are very rare and usually not available for a specific isotope ratio in a specific matrix. The best way to validate an analytical procedure in isotope analysis is to assess all influencing quantities and to set up a complete uncertainty budget (technique 4 plus 5). Preferably the calibration is performed using an isotope reference material and validation is verified by applying the analytical technique to a reference sample with a known quantity value.

\section{ISOTOPE REFERENCE MATERIALS}

Isotope reference materials usually are either pure solid elements or compounds (e.g. magnesium metal, boric acid) or mono-element solutions, which both are certified for one or more isotope amount ratio of the corresponding element. ${ }^{25)}$ Since more than 4 decades these materials have been certified by performing absolute or calibrated measurements. Such absolute measurements are realized by using synthetic isotope mixtures for the calibration of the

Table 2. Uncertainty budget of the $\delta^{11} \mathrm{~B}$ value determined for BCR-679 (White Cabbage).

\begin{tabular}{lccll}
\hline Quantity & Value & $\begin{array}{c}\text { Standard } \\
\text { uncertainty }\end{array}$ & Type A/B ${ }^{a}$ & Description of the uncertainty contribution \\
\hline$R_{\text {aliquot1 }}$ & 3.954591 & $771 \times 10^{-6}$ & $\mathrm{~A}$ & Observed ${ }^{11} \mathrm{~B} /{ }^{10} \mathrm{~B}$ ratio in processed aliquot 1 \\
$R_{\text {aliquot2 }}$ & 3.952516 & $686 \times 10^{-6}$ & $\mathrm{~A}$ & Observed ${ }^{11} \mathrm{~B} /{ }^{10} \mathrm{~B}$ ratio in processed aliquot 2 \\
$R_{\text {aliquot3 }}$ & 3.955900 & $318 \times 10^{-5}$ & $\mathrm{~A}$ & Observed ${ }^{11} \mathrm{~B} /{ }^{10} \mathrm{~B}$ ratio in processed aliquot 3 \\
$\kappa_{1}$ & 1.000000 & $410 \times 10^{-6}$ & $\mathrm{~B}$ & Sample decomposition \\
$\kappa_{2}$ & 1.000000 & $220 \times 10^{-6}$ & $\mathrm{~B}$ & Boron-matrix separation \\
$\kappa_{3}$ & 1.000000 & $110 \times 10^{-6}$ & $\mathrm{~B}$ & Procedure blank \\
$R_{\mathrm{NBS} 951}$ & 4.050898 & $116 \times 10^{-6}$ & $\mathrm{~A}$ & Observed ${ }^{11} \mathrm{~B} /{ }^{10} \mathrm{~B}$ ratio for NIST SRM 951 \\
$\kappa_{4}$ & 1.000000 & $200 \times 10^{-6}$ & $\mathrm{~B}$ & Potential inhomogeneity of NIST SRM 951 \\
$\delta^{11} \mathrm{~B}$ & -23.84 & 0.58 & $\mathrm{~B}$ & Final $\delta^{11} \mathrm{~B}$ value for BCR-679 in per mill \\
\hline
\end{tabular}

${ }^{a}$ Type A: uncertainty derived from observation (distribution of measurements values). Type B: uncertainty derived by other means than observation (e.g. certificate, expert judgement etc.).

${ }^{b}$ Index gives the fraction of each influence quantity at the total uncertainty.

Adapted with permission from ref. 11. Copyright 2011 American Chemical Society. 
applied mass spectrometer (Fig. 1). This technique requires highly enriched and highly purified isotopes. The production of such isotopes, however, is labour and money intensive. The whole process of producing synthetic isotope mixtures requires excellent preparative work and attention must be paid to any source of error. Therefore only a very limited number of laboratories work in this field. In addition to the described drawbacks the achievable standard uncertainties $(k=1)$ for the certified isotope amount ratios are currently limited to values between $0.01 \%$ and $0.001 \%$, which is larger than the precision obtained with current multi-collector mass spectrometers ${ }^{25,44)}$ (Table 1).

To bypass this uncertainty problem and potential homogeneity issues with solid materials, so-called $\delta$-reference materials in solution have been suggested by a number of scientists for all elements. ${ }^{25,44-46)}$ Elemental Solutions in principle can be produced with a homogeneity of better than $0.001 \%$, expressed as relative standard deviation. This homogeneity can be checked by using the most precise analytical procedure for carrying out repeated measurements for the isotope ratio of interest in on a substantial number of units of the candidate material.

Since decades $\delta$-reference materials are successfully used for the classical stable isotopes $(\mathrm{H}, \mathrm{C}, \mathrm{N}, \mathrm{O}, \mathrm{S})$. The determination of $\delta$-values can be carried out with lower uncertainties than the determination of isotope amount ratios, because the sample isotope ratio is related to the standard isotope ratio and most corrections cancel down (see Eq. (1)). This is also reflected in $\delta$-reference materials, where lower uncertainties can be achieved compared to isotope reference materials certified for isotope amount ratios.

For most elements, however, either suitable isotope reference materials or $\delta$-reference materials are lacking. To cover this demand and to enable traceable and comparable isotope data a tight cooperation between producers and users of isotope or $\delta$-reference materials is required.

For uranium and plutonium suitable isotope reference materials are available in a sufficient number, because in this case strong economic and sociopolitical interests play an important role due to nuclear applications. Beside these elements, boron is the only element, where the situation concerning isotope reference materials, $\delta$-reference materials and quality control materials is excellent (Fig. 2). Recently, BAM Federal Institute for Materials Research and Testing released a set of $3 \delta$-reference materials (ERM-AE120, -AE121, -AE122) nearly covering the whole range of the natural boron isotope variation. ${ }^{8)}$ This $\delta$-reference materials offer standard uncertainties $(k=1)$ of $0.3 \%$ and offer the possibility to validate analytical procedures for $\delta^{11} \mathrm{~B}$ determination at this level of uncertainty by using this materials as quality control samples. More details can be obtained from ref. 8. Several years before a set of ${ }^{10} \mathrm{~B}$-enriched isotope reference materials (ERM-AE101, -AE102, -AE103 and -AE104) has been released, which has been partly replaced by ERM-AE102a and -AE104a and which will be expanded in early 2013 by ERM-AE123 (natural isotope composition) and -AE124 $\left({ }^{10} \mathrm{~B}\right.$ enriched spike material). Thus the range between natural boron and highly enriched ${ }^{10} \mathrm{~B}$ is completely covered with isotope reference materials. Additionally, a large number of quality control and matrix reference materials covering the natural isotopic range is available from different sources. Consequently, boron can be regarded as perfect example for isotope and $\delta$-reference materials and may serve as model/prototype for other elements.

\section{Boron isotope reference and quality control materials}

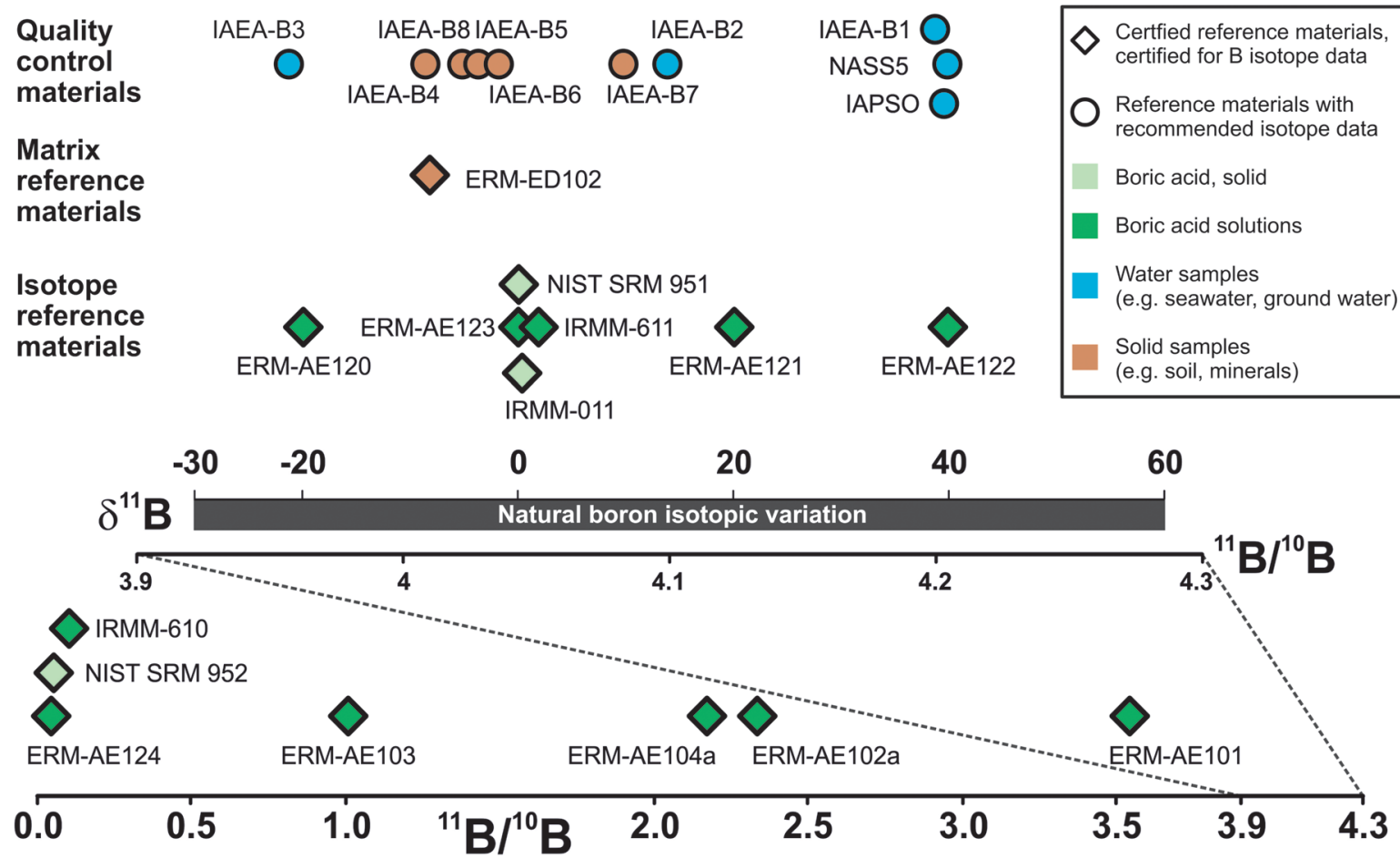

Fig. 2. Current situation for boron regarding isotope reference materials. Copied with permission from ref. 44. Copyright Springer Verlag, 2012. 


\section{CONCLUSION AND OUTLOOK}

The current generation of multi-collector mass spectrometers guarantees isotope ratio precision at the $0.001 \%$ and $0.0001 \%$ level for standard solutions. This performance enables the investigation of isotope variations for nearly all elements. But pure precision determined in standard solutions is no measure for measurement uncertainties, because real samples have intrinsic and varying difficulties such as interfering elements and matrix components. To eliminate these difficulties sophisticated sample preparation schemes consisting at least of a digestion and an analyte separation step are necessary. The whole analytical procedure requires a careful, step-by-step validation in order to guarantee the reliability of the procedure and the accuracy of the analytical result.

Moreover, the obtained results need to be metrologically traceable and thus metrologically comparable to enable the joint use and interpretation of datasets obtained in different studies or labs. Traceability of isotope data requires two things, first the use of an isotope reference material (for correcting mass fractionation) or an internationally accepted standard (as reference for the $\delta$-scale) and second the generation of an uncertainty budget including all calibration and input quantities.

Admittedly the situation with isotope and $\delta$-reference materials is not satisfactory, because too few materials are available offering sufficiently low uncertainties. Isotope research requires such materials and therefore more effort should be made to improve the situation. The case of boron may serve as perfect prototype for providing suitable isotope and $\delta$-reference materials for other elements in isotope research.

In ref. 25 a list of elements being currently under investigation has been published. But it can be assumed that isotope research in the future will cover all elements with 2 or more isotopes in any matrix. In the near future increased research can be assumed for elements such as V, Mo, $\mathrm{Ag}$, and $\mathrm{Sn}$. The focus here should be clearly on providing $\delta$-reference materials for these upcoming elements to anchor the corresponding $\delta$-scale and on matrix reference materials certified for isotope ratios or $\delta$-values in order to enable validation and quality control of existing analytical procedures. One example for a future $\delta$-reference material could be a seawater reference material certified for $\delta^{7} \mathrm{Li}, \delta^{11} \mathrm{~B}, \delta^{26} \mathrm{Mg}$ and others.

\section{Acknowledgement}

The author would like to thank Martin Rosner (IsoAnalysis UG) for fruitful discussions and for proofreading.

\section{REFERENCES}

1) J. R. De Laeter, J. K. Bohlke, P. De Bievre, H. Hidaka, H. S. Peiser, K. J. R. Rosman, P. D. P. Taylor. Atomic weights of the elements: Review 2000 (IUPAC technical report). Pure Appl. Chem. 75: 683-799, 2003.

2) B. B. Hanan, J. Blichert-Toft, D. G. Pyle, D. M. Christie. Contrasting origins of the upper mantle revealed by hafnium and lead isotopes from the Southeast Indian Ridge. Nature 432:
91-94, 2004.

3) M. Betti. Isotope ratio measurements by secondary ion mass spectrometry (SIMS) and glow discharge mass spectrometry (GDMS). Int. J. Mass Spectrom. 242: 169-182, 2005.

4) L. Balcaen, L. Moens, F. Vanhaecke. Determination of isotope ratios of metals (and metalloids) by means of inductively coupled plasma-mass spectrometry for provenancing purposes-A review. Spectrochim. Acta, B At. Spectrosc. 65: 769-786, 2010.

5) H. Pfeffer, F. Dannel, V. Römheld. Boron compartmentation in roots of sunflower plants of different boron status: A study using the stable isotopes ${ }^{10} \mathrm{~B}$ and ${ }^{11} \mathrm{~B}$ adopting two independent approaches. Physiol. Plant. 113: 346-351, 2001.

6) A. Rossmann. Determination of stable isotope ratios in food analysis. Food Rev. Int. 17: 347-381, 2001.

7) G. Fortunato, K. Mumic, S. Wunderli, L. Pillonel, J. O. Bosset, G. Gremaud. Application of strontium isotope abundance ratios measured by MC-ICP-MS for food authentication. J. Anal. At. Spectrom. 19: 227-234, 2004.

8) J. Vogl, M. Rosner. Production and certification of a unique set of isotope and delta reference materials for boron isotope determination in geochemical, environmental and industrial materials. Geostand. Geoanal. Res. 36: 161-175, 2012.

9) M. R. Palmer, P. N. Pearson, S. J. Cobb. Reconstructing past ocean pH-depth profiles. Science 282: 1468-1471, 1998.

10) A. Vengosh, K. G. Heumann, S. Juraske, R. Kasher. Boron isotope application for tracing sources of contamination in groundwater. Environ. Sci. Technol. 28: 1968-1974, 1994.

11) M. Rosner, W. Pritzkow, J. Vogl, S. Voerkelius. Development and validation of a method to determine the boron isotopic composition of crop plants. Anal. Chem. 83: 2562-2568, 2011.

12) T. Bohn, T. Walczyk, L. Davidsson, W. Pritzkow, P. Klingbeil, J. Vogl, R. F. Hurrell. Comparison of urinary monitoring, faecal monitoring and erythrocyte analysis of stable isotope labels to determine magnesium absorption in human subjects. Br. J. Nutr. 91: 113-120, 2004

13) S. F. Boulyga. Calcium isotope analysis by mass spectrometry. Mass Spectrom. Rev. 29: 685-716, 2010.

14) T. Walczyk, F. von Blanckenburg. Natural iron isotope variations in human blood. Science 295: 2065-2066, 2002.

15) K. G. Heumann, S. M. Gallus, G. Radlinger, J. Vogl. Accurate determination of element species by on-line coupling of chromatographic systems with ICP-MS using isotope dilution technique. Spectrochim. Acta, B At. Spectrosc. 53: 273-287, 1998.

16) J. Vogl, W. Pritzkow. Isotope dilution mass spectrometry-A primary method of measurement and its role for RM certification. Mapan 25: 135-164, 2010.

17) I. T. Platzner. Modern Isotope Ratio Mass Spectrometry, John Wiley \& Sons, Chichester, 1997.

18) F. Vanhaecke, L. Balcaen, D. Malinovsky. Use of single-collector and multi-collector ICP-mass spectrometry for isotopic analysis. J. Anal. At. Spectrom. 24: 863-886, 2009.

19) J. Vogl. in ICP Mass Spectrometry (Ed: S. Nelms), Blackwell Publishing Ltd., Oxford, 2005, p. 147.

20) J. F. Rudge, B. C. Reynolds, B. Bourdon. The double spike toolbox. Chem. Geol. 265: 420-431, 2009.

21) A. O. Nier. A redetermination of the relative abundances of the isotopes of carbon, nitrogen, oxygen, argon, and potassium. Phys. Rev. 77: 789-793, 1950.

22) J. Meija. Calibration of isotope amount ratios by analysis of isotope mixtures. Anal. Bioanal. Chem. 403: 2071-2076, 2012.

23) S. Epstein, T. Mayeda. Variation of O-18 content of waters from natural sources. Geochim. Cosmochim. Acta 4: 213-224, 1953.

24) T. B. Coplen. Guidelines and recommended terms for expression of stable-isotope-ratio and gas-ratio measurement results. Rapid Commun. Mass Spectrom. 25: 2538-2560, 2011.

25) J. Vogl, W. Pritzkow. Isotope reference materials for present and future isotope research. J. Anal. At. Spectrom. 25: 923-932, 2010

26) S. Richter, H. Kuhn, Y. Aregbe, M. Hedberg, J. Horta-Domenech, 
K. Mayer, E. Zuleger, S. Burger, S. Boulyga, A. Kopf, J. Poths, $\mathrm{K}$. Mathew. Improvements in routine uranium isotope ratio measurements using the modified total evaporation method for multi-collector thermal ionization mass spectrometry. J. Anal. At. Spectrom. 26: 550-564, 2011.

27) $\mathrm{Nu}$ Instruments. Nu Plasma II Application Note 13 and $\mathrm{Nu}$ Plasma II Specifications issue 1.8, http://www.nu-ins.com/index. php/downloads (last accessed 28.08.2012)

28) Thermo Fisher Scientific, Neptune Plus Application Notes 30158, 30187 and Neptune plus Specifications 2011; http://www.thermoscientific.com/ecomm/servlet/productsdetail_11152_L10565_ 98065_11961870_-1 (last accessed 28.08.2012)

29) IsotopX. Limited, Phoenix Nuclear Product Description and Specifications, V1.3n March 2011, http://www.isotopx.com/ downloads.htm (last accessed 28.08.2012)

30) Thermo Fisher Scientific, Triton Plus Application Note 30015: High Precision Strontium and Neodymium Isotope Analysis, http:/www.thermoscientific.com/ecomm/servlet/ productsdetail_11152_L10565_98065_11962736_-1, (last accessed 28.08.2012)

31) W. Pritzkow, S. Wunderli, J. Vogl, G. Fortunato. The isotope abundances and the atomic weight of cadmium by a metrological approach. Int. J. Mass Spectrom. 261: 74-85, 2007.

32) T. Hirata. in Isotopic Analysis-Fundamentals and Applications Using ICP-MS (Ed: F. Vanhaecke, P. Degryse), Wiley-VCH, Weinheim, 2012, pp. 93-112.

33) V. T. C. Chang, A. Makishima, N. S. Belshaw, R. K. O’Nions. Purification of $\mathrm{Mg}$ from low-Mg biogenic carbonates for isotope ratio determination using multiple collector ICP-MS. J. Anal. At. Spectrom. 18: 296-301, 2003.

34) K. Moeller, R. Schoenberg, R.-B. Pedersen, D. Weiss, S. Dong. Calibration of the new certified reference materials ERM-AE633 and ERM-AE647 for copper and IRMM-3702 for zinc isotope amount ratio determinations. Geostand. Geoanal. Res. 36: 177$199,2012$.

35) A.-D. Schmitt, S. J. G. Galer, W. Abouchami. High-precision cadmium stable isotope measurements by double spike thermal ionisation mass spectrometry. J. Anal. At. Spectrom. 24: 10791088, 2009.
36) Z. Xue, M. Rehkämper, M. Schönbächler, P. J. Statham, B. J. Coles. A new methodology for precise cadmium isotope analyses of seawater. Anal. Bioanal. Chem. 402: 883-893, 2012.

37) F. Wombacher, M. Rehkämper. Problems and suggestions concerning the notation of cadmium stable isotope compositions and the use of reference materials. Geostand. Geoanal. Res. 28: 173-178, 2004.

38) International Vocabulary of Metrology-Basic and General Concepts and Associated Terms VIM. 3rd edition, JCGM 200:2008, http://www.bipm.org/en/publications/guides/vim.html also ISO Guide 99-12: 2007.

39) T. Meisel. in Isotopic Analysis-Fundamentals and Applications Using ICP-MS (Ed: F. Vanhaecke, P. Degryse), Wiley-VCH, Weinheim, 2012, p. 165.

40) M. Rosner, A. Meixner. Boron isotopic composition and concentration of ten geological reference materials. Geostand. Geoanal. Res. 28: 431-441, 2004.

41) JCGM. 100:2008, Evaluation of measurement data-Guide to the expression of uncertainty in measurement, Joint Committee for Guides in Metrology, http://www.bipm.org/en/publications/ guides/gum.html or ISO/IEC Guide 98-3:2008, Uncertainty of measurement-Part 3: Guide to the expression of uncertainty in measurement, International Organization for Standardization (Geneva, Switzerland).

42) S. L. R. Ellison, A. Williams (Eds.). Eurachem/CITAC Guide: Quantifying Uncertainty in Analytical Measurement, 3 Edition, 2012. Available from www.eurachem.org

43) ISO 17025: 2005, General requirements for the competence of testing and calibration laboratories, International Organization for Standardization (Geneva, Switzerland).

44) J. Vogl, M. Rosner, W. Pritzkow. The need for new isotope reference materials. Anal. Bioanal. Chem. 405: 2763-2770, 2013.

45) R. D. Vocke, J. L. Mann. Isotopic reference materials: New frontiers leading to new opportunities. Geochim. Cosmochim. Acta 74: A1084, 2010.

46) E. Ponzevera, C. R. Quetel, F. Hendrickx, G. Fortunato. Production and certification of candidate $\mathrm{Pb} \delta$-iCRMs ERM-3800 and ERM-3810 series. J. Anal. At. Spectrom. 24: 402-406, 2009. 